\title{
Friend Recommendation System for Online Social Networks
}

\author{
Dagadu M. Jadhavar \\ Computer Science and Information Technology \\ MBES College Of Engineering, Ambajogai, India
}

\author{
V. R. Chirchi \\ Computer Science and Information Technology \\ MBES College Of Engineering, Ambajogai, India
}

\begin{abstract}
The Already social networking services recommends friends list to requesting user that is based on their social graphs, but they cannot fulfill user need to user preferences on friend selection. In this paper, we represent Friend recommendation, as lifestyle based friend recommendation system for social networks. Collecting data from smartphone sensors and identifying lifestyle of user, if lifestyle having high score similarities then recommend friend to user. Here probability Distribution algorithm is used for extracting lifestyle of users learns from text mining. Also use the Activity recognition for classify the activity of user and propose a similarity metric to find the similarity of life styles between users, and after this one finding user's impact in consideration of life styles with a friend-matching graph. User request is received then friend recommendation system gives response as similar lifestyle matching friends lists to user query. At last, Friend recommendation system uses the feedback modules to improve the recommendation accuracy. We have Friend recommendation system is to implemented on the Androidbased smartphones, By using Friend recommendation system user can get best friend list for preferences of users in choosing friends.
\end{abstract}

\section{Keywords}

Life style,Friend, Bag of Activity model, Social Networks, Similarity Metric.

\section{INTRODUCTION}

Mobile Computing is technology a transfer of data, audio file ,video ,images with any other wireless devices is called mobile computing. Mobile computing contains Mobile communication, Mobile hardware, and Mobile software. Social network are website that allows people to connect with friends and make new friends, virtually, and share content, interact, create community of similar interest, working, reading, friendship, relationship, relationships commercial etc. The currently, people made friends with other people are who live or work closest to themselves, like neighbor's or colleges. The current social services such as Facebook, twitter, LinkedIn [2]

To group people by using following's things are habits or lifestyle, Attitudes, Tastes, economic Level, and People already known. The already services depends on pre-existing user relationship to choose friend user. Example. Facebook works such as choosing those who already share common friends and recommends similar people as friends. Facebook also analysis of people depends on tastes, already known People Location based, Education etc. But these social services cannot give best friend to user. Facebook to find out required friend it much takes lot of time with current services .The already existing services are not available new service such as habits or life styles related friend group is required.
We can take the challenge to prepare the friend book based on life style and habits. Lifestyle is related to user daily routines and activities. Activates means action performed by people such as college day or exam day or exercise etc. If we consider exercise having life style contains walking, running activity, also contain standing or sitting activity etc. Probabilistic topic model uses text mining that defined as document(Life document or daily lives) is mixture of topics(Lifestyle e.g. Exercise).Topics is combination of Words(activities e.g. Running) Smartphone (e.g. iPhone or android based smartphone)are set of sensors such as gyroscope ,GPS, microphone ,accelerometer and camera. Smartphone is used to collecting the lifestyle or real time daily routines of users [1]

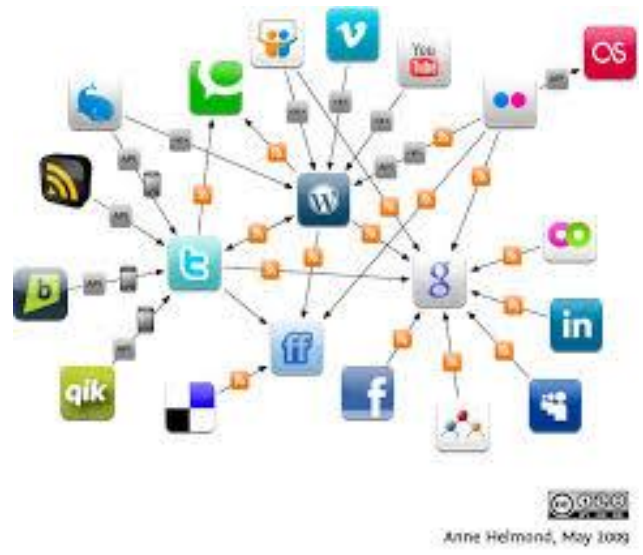

Fig 1: Social networking Services

A. Multiple Challenges:

- How to automatically and accurately identifying lifestyle from noisy and heterogeneous data.

- How to measure similarity of user by considering lifestyle

- Who can recommend to the user among all friend users?

B. Contributions or Implementation steps:

- In friend book any user forward the user query related to life style. Friend book provide the service same life styles users as a recommendation users

- Smartphones discussions and daily activities we can collect here

- Life style analysis identifies similar users.

- Development \& Implementation of probability distribution to find the user with similar interest and recommend friend to user. 
- Friend matching graph

- How many users are available as a matching users count here using probabilistic model

- Generate the index list

- Query: friend recommendation list.

- After display recommendation list: feedback to server.

\section{LITERATURE REVIEW}

Friend recommendation scheme to refer some already paper that are useful us to some are explaining as follows:

- 2006: A. S. Pentland and N. Eagle." Reality Mining: Sensing Complex Cocial Systems". Personal Ubiquitous Computing, 10(4):255-268, To discover daily location driven routines from large scale location data [16].

- 2007: N. D. Lane, and E. Miluzzo,A. T. Campbell S. B. Eisenman,. "Cenceme-Injecting Sensing Presence into Social Networking Applications". Proc. of EuroSSC, pages 1-28,.It is used multiple sensors on the smartphone to capture user's activities, state, habits and surroundings [17]. 2008:T. Huynh, B. Schiel and M. Fritz. "Discovery of Activity Patterns using Topic Models. Proc. of Ubi Comp." Develop an unsupervised methodology based on two differing probabilistic topic models and apply them to the daily life. The resulting distributions of words for latent topics, as well as topics given days, and topics given users, re-veal hidden structure of routines which use to perform varying tasks, including finding users or groups of users that display given routines, and determining times [4].

- 2010: "Darwin Phones: the Evolution of Sensing and Inference on Mobile Phones". Proc. of MobiSys, pp. 5-20, C. T. Cornelius,A E. Miluzzo, A. Ramaswamy, Liu and A. T. Campbell, T. Choudhury, Z.. Ramaswamy, T. Choudhury, and Sound Sense used microphone on smartphone to recognize general sound types (e.g., music, voice) and discover user specific sound events [6].

- 2010:K. Farrahi and D. Gatica-Perez. "Probabilistic mining of socio-geographic routines from mobile phone data". Selected Topics in Signal Processing, IEEE Journal of, Vol. 4, No. 4, pp. 746-755, investigate probabilistic topic models as unsupervised machine learning tools for large-scale socio-geographic activity mining. They propose a methodology based on probability distribution Algorithm for the discovery of dominant location routines. In this paper they used two Probalistic model namely Multi-Level Topic Model and Pair wise -Distance Topic Model. First they propose a Multi-Level Topic Model as a method to incorporate multiple time duration sequences into a probabilistic generative topic model. And then they propose the Pair wise-Distance Topic Model as an approach to address the problem of modeling long duration activities with topics. Overall, this thesis addresses Investigations principled on mathematical models and multiple types of mobile phone sensor data are performed to mine real life human activities in largescale scenarios. [5].

- 2011:L. H. Holtzman, Bian. "Online friend recommendation through personality matching and collaborative filtering". Proc. of UBICOMM, pages 230235.Present Matchmaker, a collaborative filtering friend recommendation system based on personality matching.
The goal of Matchmaker is to leverage the social information and mutual understanding among people in existing social network connections, and produce friend recommendations based on rich contextual data from people's physical world interactions. Matchmaker allows users' network to match them with similar TV characters, and uses relationships in the TV programs as parallel comparison matrix to suggest to the users friends. The system's ranking schema allows progressive improvement on the personality matching consensus and more diverse branching of users' social network connections.[3]

\section{PROPOSED SYSTEM}

Friend recommends system is first social service that recommends friends depends on user query i.e. Habits or collecting the Lifestyle information of other user identified based on smartphone sensors. Friend recommendation system is client-server mode. Client is each smartphone used by user, server is Database or clouds.

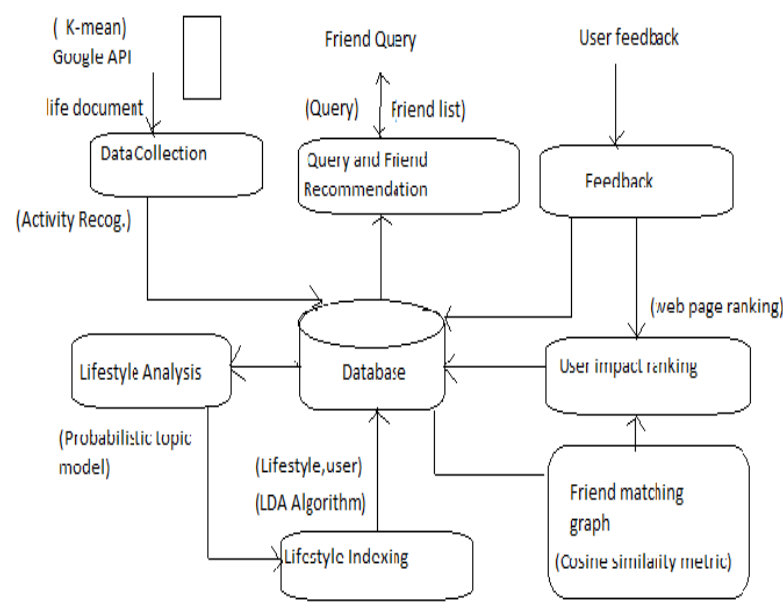

Fig 2: Abstract Diagram for Friend Recommend system

\subsection{Client side}

Each smartphone can collect information of its user, perform real-time activity recognition and generated report life documents to the servers.

\subsubsection{Registration Module}

In this Module First user can register to friend recommend system by adding information of first name, surname, Email ID, password, and birthdate and submit .All data are saved to database and generate key for user by admin and gives access to friend Recommendation system. Contains user register, search user, send request, view request, accept request, view rank etc.

\subsection{Server Side}

It contains seven modules that perform the task of friend recommendation

\subsubsection{Admin}

Admin having all rights of friend Recommendation system, it can add new user, collect data from user name, photo, and lifestyle and also collect data from Google API. Add new user, add detail, view detail, add group, view group, form group, view query, view user rank, view friend matching, view android user, view feedback, logout. 


\subsubsection{Data Collection Modules}

Collect activity of each users from smartphones, etc. This life document is used to matching the friends for recommending to the user request, Measure probability of lifestyle of user. In probabilistic topic model denote the frequency of word as a entropy variances. We use the bag-ofactivity model is used to replace sequence in life document with their no of time word or activity occur in it with indicating frequency. Life style indexing put life style in form of (lifestyle, users) of user and store in database. [1]

\subsubsection{Life Style Analysis}

Here, we have necessary to group or classify or recognize user activity. Two type of motion sensor in smartphone accelerometer and gyroscope for taking lifestyle of user motion. We can use k-mean algorithm unsupervised learning for group data. Activity recognition Perform takes raw data collected form user smartphone i.e. noise data, noise data is filtered with sliding windos.by preprocessing. Then preprocessed data used to feature extraction by using correlation, standard division, mean, for generate Feature vectors. Then make similar activity group for used to lifestyle matching of user. The collected data is gives to LDA algorithm for lifestyle extraction and show similar word in certain topic of document [1]

\subsubsection{Life style indexing}

Use reverse indexing for indexing life style, it working of indexing is instead of user life style index shows the user1 having how much life style, similar user 2 how much total lifestyle to identified. Reverse indexing takes the every lifestyle having how much users occur for particular lifestyle. Reverse indexing takes the user lifestyle put into database in format of (lifestyle, users) .

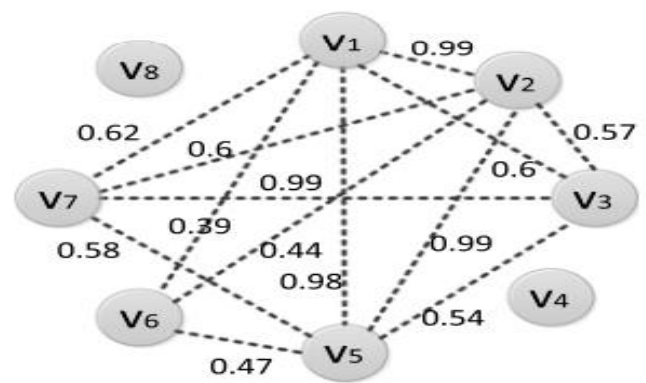

Fig 3: Friend Matching Graph.

\subsubsection{Friend matching graph modules}

It shows similarity lifestyle matching of user by using weighted undirected graph. Graph containing vertices is the user and edge with its weight between users means similar lifestyle. If user is similar life style that means similarity is greater than similarity threshold is predefined constant and similarity weight is greater than 60 percent. If some vertices are not linking edge means no similarity.

But in this paper, we recommend friends depend on lifestyle similarity or habits extracted by using probabilistic topic model. Also provide high impact ranking users and gives the high preferences to choosing friends from potential friend list. Friend recommendation gives feedback facility to user gives their feedback for their need is meet or not. Feedback can be increase the accuracy and ranking.

\section{FUTURE WORK}

Main issue in front of friend recommendation system is similarity threshold used for friend matching graph is constant in our current prototype friend recommendation system.

\section{REFERENCES}

[1] Zhibo Wang ,Student member, IEEE, Jilong Liao, Qing Cao, Member, IEEE, Hairong Qi, Senior Member ,IEEE and Zhi Wang ,Member, IEEE" FriendBook:A Semantic Based Friend Recommendation System for Social Networks" EEE Transaction on mobile computing, VOL.14,No.3,MARCH 2015.

[2] Facebookstatistics. http://www.digitalbuzzblog.com/ facebook-statistics-stats-facts-2011/.

[3] L. Bian and H. Holtzman. Online friend recommendation through personality matching and collaborative filtering. Proc. of UBICOMM, pages 230-235, 2011.

[4] T. Huynh, M. Fritz and B. Schiel. Discovery of Activity Patterns using Topic Models. Proc. of Ubi Comp. 2008.

[5] K. Farrahi and D. Gatica-Perez. Probabilistic mining of socio-geographic routines from mobile phone data. Selected Topics in Signal Processing, IEEE Journal of, Vol. 4, No. 4, pp. 746-755, 2010.

[6] C. M. Bishop. Pattern recognition and machine learning. Springer New York, 2006.

[7] P. Desikan, N. Pathak, J. Srivastava, and V. Kumar Incremental page rank computation on evolving graphs. Proc. of WWW, pages 1094-1095, 2005.

[8] J. Kwon and S. Kim. Friend recommendation method using physical and social context. International Journal of Computer Science and Network Security, 10(11):116$120,2010$.

[9] E. Miluzzo, N. D. Lane, S. B. Eisenman, and A. T. Campbell. Cenceme-Injecting Sensing Presence into Social Networking Applications. Proc. of EuroSSC, pages 1-28, October 2007.

[10] Y. Zheng, Y. Chen, Q. Li, X. Xie, and W.-Y. Ma. Understanding Transportation Modes Based on GPS Data for Web Applications. ACM Transactions on the Web (TWEB), 4(1):1-36, 2010.

[11] J. Biagioni, T. Gerlich, T. Merrifield, and J. Eriksson. EasyTracker: Automatic Transit Tracking, Mapping, and Arrival Time Prediction Using Smartphones. Proc. of SenSys, pages 68-81, 2011. 